\title{
Chiral Symmetry Breaking during Crystallization: An Advection-Mediated Nonlinear Autocatalytic Process
}

\author{
Julyan H. E. Cartwright, ${ }^{1}$ Juan Manuel García-Ruiz, ${ }^{1}$ Oreste Piro, ${ }^{2}$ C. Ignacio Sainz-Díaz, ${ }^{1}$ and Idan Tuval ${ }^{2}$ \\ ${ }^{1}$ Laboratorio de Estudios Cristalográficos, CSIC, E-18100 Armilla, Granada, Spain \\ ${ }^{2}$ Institut Mediterrani d'Estudis Avançats, CSIC-UIB, E-07122 Palma de Mallorca, Spain
}

(Received 8 March 2004; published 13 July 2004)

\begin{abstract}
When a chiral chemical compound crystallizes from solution or from its melt, stirring often results in the formation of crystals of just one of the two possible enantiomers, while without fluid advection both enantiomers are formed. We demonstrate with simulations of the dynamics of the system that secondary nucleation is a nonlinear autocatalytic phenomenon that can explain these observations. Furthermore, we present theoretical arguments and experimental results that suggest that at the microscale the mechanism of secondary nucleation is whisker crystal growth and dispersion in the fluid flow.
\end{abstract}

PACS numbers: $81.10 . \mathrm{Aj}, 47.52 .+\mathrm{j}, 47.55 . \mathrm{Kf}, 68.70 .+\mathrm{w}$

A century and a half ago, Pasteur [1] discovered that inorganically synthesized tartaric acid differs from that obtained from plants in crystallizing as two mirrorimage versions, or enantiomers, whereas only one of the two forms is found in biologically derived samples. This symmetry is broken in all biological systems, as amino acids, the building blocks of proteins, and thence of every living organism, are found in nature almost exclusively as the laevo (left-handed) enantiomers, while natural sugars are all dextro (right handed). However, inorganic chemical reactions involving chiral products commonly yield a racemic mixture of both enantiomers [2]. So it came as a considerable surprise when a decade ago it was shown that simply stirring during the crystallization of sodium chlorate from solution was sufficient to produce a yield approaching $100 \%$ of just one enantiomer [3]. Whereas under normal conditions a distribution of the proportion of one or other enantiomer obtained in a series of experiments falls on a typical Gaussian curve, with the peak yield at $50 \%$ of each enantiomer, in the stirred experiments the distribution is bimodal, with the peak yield close to $100 \%$ of one or the other enantiomer. Similar results have been obtained in stirred crystallization of an organic molecule, 1,1'-binaphthyl, from its melt [4], and in the stirred synthesis of a chiral cobalt complex [5,6]. Subsequent discussion has revolved around how this symmetry-breaking mechanism operates. The importance of the detachment of parts of the crystal surface upon contact with the stirring bar [7], and convection in the fluid [8], have both been proposed as possible mechanisms.

The purpose of this Letter is to clarify the mechanism of chiral symmetry breaking during crystallization. First, we show with numerical simulations that secondary nucleation-nucleation of new crystals caused by the presence of an existing primary crystal-is a nonlinear autocatalytic process capable of explaining the experimental results. Second, we present theoretical arguments to show that convection and mechanical crushing are on the microscale the same mechanism for secondary nucleation: detaching whiskers or other asperities from the surface of the primary crystal by supplying sufficient force to rupture the chemical bonds. Finally, we demonstrate with laboratory experiments that both sodium chlorate crystallizing from solution and 1,1'binaphthyl crystallizing from its melt produce such whisker crystals.

To nucleate a crystal requires overcoming a certain energy barrier [9]. The height of the barrier is a function of the concentration of a solution, or the temperature of a melt. A supersaturated solution, concentrated beyond its saturation point, or a supercooled melt, cooled below its freezing point, are systems metastable to nucleation. With a solid surface already present, less energy is required to surmount the barrier. Thus functions secondary nucleation, in which the presence of one crystal facilitates the production of others. A half century ago, Frank [10] suggested that a form of autocatalysis in which each enantiomer catalyzes its own production, while suppressing that of its mirror image, might have nonlinear dynamics leading to the amplification of small initial fluctuations in the concentrations of the enantiomers. Secondary nucleation can act as just such a nonlinear autocatalytic process, as, in the case of chiral crystallization, secondary nuclei possess the same chirality as the mother crystal, so the presence of a crystal of a given chirality catalyzes the production of further crystals with the same chirality.

To demonstrate that these ideas can explain the experimental observations, we construct a minimal model for chirality selection via secondary nucleation in solution or in the melt by building on the work on nonlinear autocatalytic processes in flows of Metcalfe and Ottino [11], to which we add a realistic but simple model for the physics of crystal nucleation and growth. We use, as they did, the chaotic journal-bearing flow to illustrate the concept. We model supersaturation or supercooling as an initially uniform distribution of passive-scalar particles in the journal-bearing flow. We allow primary nuclei to appear 
randomly at any point with a probability depending upon the supersaturation or supercooling. These are randomly assigned one of two chiralities $L$ (laevo) or $D$ (dextro). As nuclei appear they deplete their vicinity of solute or increase the local temperature, so the supersaturation or supercooling in the fluid around them decreases. The nuclei move with the flow and accumulate putative secondary nuclei on their surfaces at a rate depending on the supersaturation or supercooling. These have the same chirality as their parent, and are shed into the flow at a rate depending on the shear stress at their position. With this model we can investigate how the ratio $L / D$ depends on the advection rate and on the initial supersaturation or supercooling, and can evaluate the relative contributions of primary versus secondary nucleation.

Metcalfe and Ottino investigated a model in which there are two competing reaction pathways, but in which there is neither primary nor secondary nucleation. Starting from two initial seeds, one of each chirality, they observed the dominance of one of the competing chiralities upon the advection of the seeds in the flow. But if we allow in the Metcalfe and Ottino model the random introduction - as in primary nucleation-of seeds of both enantiomers, there is no longer chiral selection, and the final result is that there is $50 \%$ of each enantiomer. In Fig. 1 we present simulations with black dots representing the uniformly distributed supersaturation or supercooling, and red and green dots, which are crystal nuclei of different chiralities. We may introduce the enantio-
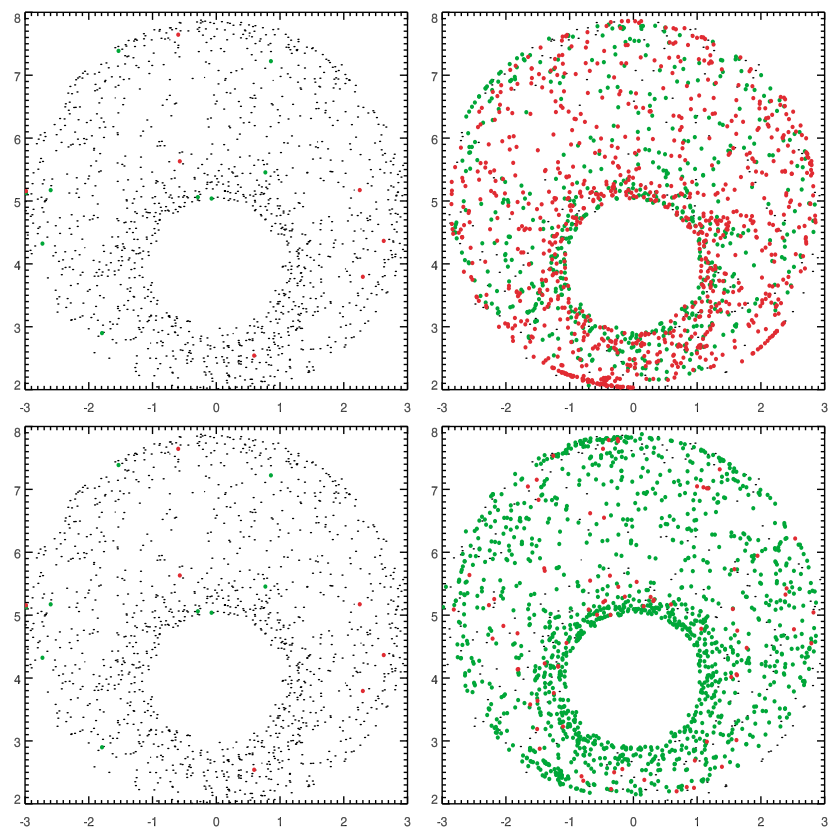

FIG. 1 (color). Simulations of (top) the Metcalfe and Ottino model with primary nucleation and (bottom) our model, demonstrating the importance of secondary nucleation for the production of an enantiomeric excess. Initial conditions are shown on the left; on the right are the final states after seven periods of the flow. meric excess $\varepsilon=\left(n_{D}-n_{L}\right) /\left(n_{D}+n_{L}\right)$, where $n_{D}$ and $n_{L}$ are the numbers of crystals of each enantiomer, as a means to quantify the symmetry breaking. In the two upper plots we have the Metcalfe and Ottino model with primary nucleation. Initially there is $50 \%$ of each enantiomer, and after many flow periods there is still $50 \%$ of each $(\varepsilon=0.001)$. But if we add the production and growth of secondary nuclei to the model, and their detachment from the mother nucleus and dispersal when they pass through a region of high shear, the results change dramatically, as we display in the two lower plots. At first, as before, there is no enantiomeric excess, but by the end we observe an overwhelming predominance of the green enantiomer, corresponding to a value of $\varepsilon$ of 0.899 .

Thus the addition of primary nucleation to the Metcalfe and Ottino model restores enantiomeric parity, and secondary nucleation can reintroduce symmetry breaking. In Fig. 2, we plot the enantiomeric excess with our model against the rate of introduction of primary nuclei (the nucleation flux, controlled experimentally by the supersaturation or supercooling), and against the advection rate, upon which secondary nucleation depends [12]. We see that, even for high supersaturation or supercooling, when primary nucleation has the most influence, secondary nucleation can overwhelm primary nucleation for fast enough advection and produce a large enantiomeric excess. This accounts for some

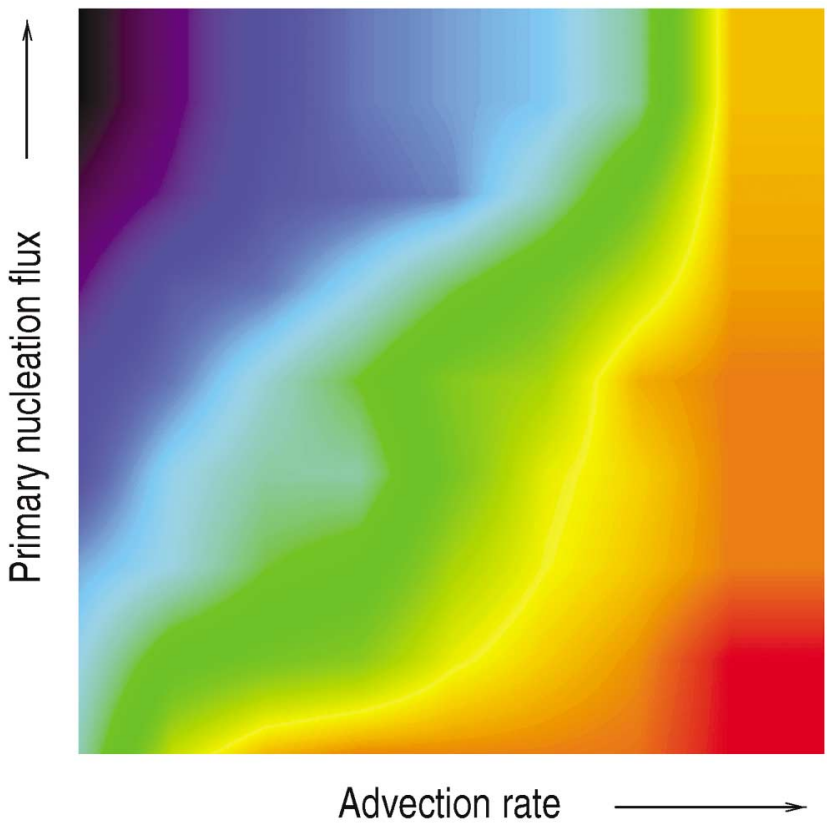

FIG. 2 (color). Plot of absolute value of enantiomeric excess $|\varepsilon|$ for our model against the rate of introduction of primary nuclei (nucleation flux, defined as the fraction of primary crystals produced per rotation cycle, in the range [0.01, 0.5]) and against advection rate (parametrized by the total angle of rotation $\theta \in[4 \pi, 12 \pi]$ ). The color scale is as a rainbow, with red representing $|\varepsilon|=1$, and black $|\varepsilon|=0$. 
recent experimental results showing chiral symmetry breaking in stirred crystallization of sodium chlorate at very high supersaturations [13].

We have shown with these simulations that secondary nucleation can explain the earlier experimental results, but by what means is it operating? Secondary nucleation has been extensively investigated, particularly for its relevance to industrial crystallization [14,15]. The mechanism on the microscale of homochiral secondary nucleation must presumably involve the surface of the mother crystal. Contact nucleation, in which collisions between one crystal and another, or between a crystal and the fluid boundaries (container walls, stirring bar, etc.) break pieces off the surface, and fluid shear, which may also detach fragments from the crystal surface, have each been put forward as responsible for homochiral secondary nucleation $[7,8]$. Both of these mechanisms involve the idea of the production and subsequent removal of relatively weakly attached homochiral material from the crystal surface. Other forms of secondary nucleation operate without regard to chirality. These involve crystal embryos (prenuclei) in the fluid adjacent to the mother crystal, which, in chiral crystallization, may be of either chirality. These achiral mechanisms may well be important, especially at high supersaturations or supercoolings at which crystal embryos in the fluid are more numerous [16]. Here, however, we are interested in understanding the dynamics of homochiral secondary nucleation.

For either mechanical or fluid forces to break material off from the growing crystal surface, that material must be relatively weakly attached, which implies that the surface must be rough, rather than smooth. While crystals at low supersaturations or supercoolings grow by the addition of material at the edges of smooth layers (socalled tangential growth), at increasing supersaturations or supercoolings the surface grows in a more disordered manner (normal growth) [17]. It is clear that the larger the number and size of asperities on the crystal surface, the greater the probability of detaching fragments by mechanical shock or fluid shear. The detached pieces possess the same chirality as the mother crystal of which they previously formed a part. At the highest supersaturations or supercoolings, these asperities can be the result of normal growth, but even with tangential growth at more moderate supersaturations or supercoolings it is possible to have structures that are easily detached. Whisker crystals are an example. In this crystal-growth morphology, long thin crystallites, or whiskers (defined as crystals with aspect ratio greater than 10:1), grow out from the substrate beneath.

It is not hard to see how whisker crystals may cause secondary nucleation, as their shape makes them easy to detach from the primary crystal. Let us estimate the force that the fluid flow or contact with a stirring bar or the wall could impart, and compare it with the force needed to break off a whisker. Consider a cuboidal whisker $b$ meters long and $a$ meters in its other two dimensions, giving it an aspect ratio $R=b / a$. Assume the whisker to be a flat plate of surface area $a b \mathrm{~m}^{2}$ orthogonal to a fluid flow of $v=1 \mathrm{~m} \mathrm{~s}^{-1}$. The force exerted on the whisker by the fluid is the mass of fluid deflected per second $\rho a b v(\rho=$ $10^{3} \mathrm{~kg} \mathrm{~m}^{-3}$ is the fluid density) multiplied by its velocity $v$. As the whisker is a cantilever projecting from the crystal surface, the force acting to open a crack at its base is the above force multiplied by the mechanical advantage $R$, which gives us an estimate $F_{f}=\rho b^{2} v^{2}=$ $10^{3} b^{2} \mathrm{~N}$. If, on the other hand, we consider the force imparted to the whisker by its mother crystal of mass $M=2 \times 10^{-3} \mathrm{~kg}$ hitting the wall or the stirring bar and decelerating from the fluid velocity $v$ to zero in the length $b$ of the whisker, we obtain $M v^{2} /(2 b)$, which again should be multiplied by the mechanical advantage $R$ to give $F_{w}=M v^{2} /(2 a)=10^{3} / a \mathrm{~N}$. Let us compare these estimates with the force needed to break off a whisker. This breakage will preferentially occur at its base, where, as indicated above, the loading is the greatest. The whisker has a cross-sectional area of $a^{2} \mathrm{~m}^{2}$. A cross section then contains of order $10^{18} a^{2}$ atoms (assuming $10^{9}$ atoms $\mathrm{m}^{-1}$ ). Let us assume that each possesses a single chemical bond linking it to the mother crystal below. Then breaking off the whisker consists in rupturing $10^{18} a^{2}$ bonds, each of which requires of order $10^{-8} \mathrm{~N}$. This gives the total force required as $F_{b}=10^{10} a^{2} \mathrm{~N}$. This rough estimate assumes that the whisker crystal is defect-free, and, in fact, the figure arrived at is rather close to much more sophisticated calculations of the theoretical maximum strength of defect-free materials [18]. Although the whisker itself is likely to be without defects, it may be attached to the mother crystal by a dislocation, which would lower the strength by several orders of magnitude [18] and make it easier to snap off at its base. In any case, we can now observe the relative importance of fluid and mechanical forces on the whisker: $F_{f} / F_{w}=a b^{2}$, which is normally considerably less than unity; the fluid forces are weaker. However, $F_{f} / F_{b}=R^{2} / 10^{7}$, so fluid forces can detach a whisker with aspect ratio $R>10^{7 / 2}$, which appears quite plausible. The stronger mechanical forces can break off any sufficiently small whisker when $F_{w} / F_{b}=10^{-7} / a^{3}>$ 1 , implying $a<10^{-7 / 3} \mathrm{~m}$. Both fluid and mechanical forces appear then to be eminently capable of breaking off whiskers during stirred crystallization.

Sodium chlorate is an achiral ionic compound that crystallizes in the cubic space group $P 2_{1} 3$ as two chiral forms. Its crystallization has been much studied, and it is with sodium chlorate that the phenomenon of the selective crystallization of one enantiomer with stirring was first seen [3]. Its propensity to produce whisker crystals has been noted $[19,20]$. We have crystallized sodium chlorate from solution and have observed the morphology of the crystals with scanning electron miscroscopy. As we see in Fig. 3, whisker crystals are notable features of the crystal surface at higher supersaturations. An interesting study has shown growth hillocks on the faces of solution-grown sodium chlorate crystals [21]. Many of 


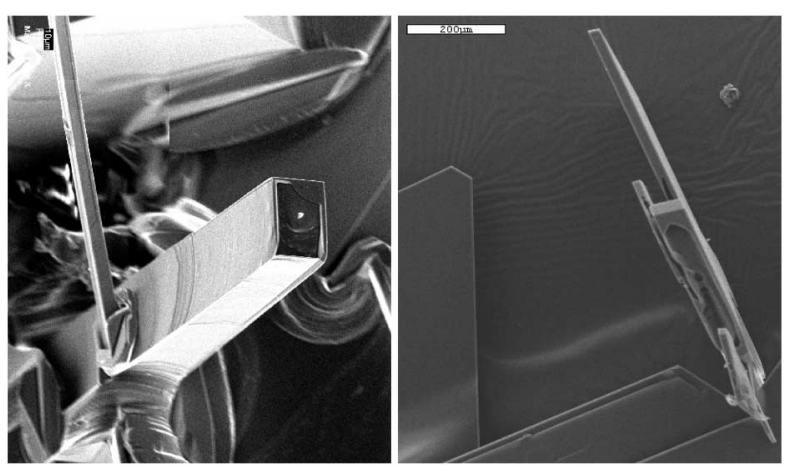

FIG. 3. Whiskers of (left) sodium chlorate crystallized from solution, and (right) 1,1'-binaphthyl crystallized from its melt.

these resulted from dislocation defects in the substrate crystal structure. These may be incipient whiskers, and such defects at the base would make them easier to break off.

We wished to see whether whisker crystal growth may be a general mechanism capable of explaining similar results with different compounds that show the same enantiomer selection behavior on crystallization. We chose 1,1'-binaphthyl, an organic molecule that when crystallized from its melt accompanied by stirring shows similar enantiomer selectivity to sodium chlorate [4]. It differs from sodium chlorate in that its chirality is present at the molecular level: each molecule of 1,1'binaphthyl possesses a chemical bond with restricted rotation, so below a certain critical temperature there are two enantiomeric forms of the molecule. Hence, sodium chlorate is achiral before crystallization, as it exists in solution as more or less dissociated ions or clusters without a fixed chirality, but forms a chiral crystal, while 1,1'-binaphthyl exists in the melt below a certain critical temperature as chiral molecules that form a chiral crystal. We crystallized 1,1'binaphthyl from the melt. In this case too, whisker crystals growing out from the primary crystal feature prominently; see Fig. 3. The presence of whiskers in these two disparate systems strengthens the idea that whisker growth is a common mechanism for secondary nucleation. Although we have shown the existence of large (mesoscale) whiskers in Fig. 3, if these exist, ergo smaller whiskers must too, down to incipient whiskers on the molecular scale.

We have been discussing chiral symmetry breaking during stirred crystallization. However, the mechanism at work in these experiments is not stirring per se, but is the formation and dispersion of homochiral nuclei throughout the fluid by flow produced by whatever means. The direction of stirring is not important here. With sodium chlorate, an enantiomeric excess can sometimes be obtained in unstirred solutions too, as natural convection caused by concentration or temperature differences within the solution can play the role of stirring (forced convection) to propagate secondary nuclei [22]. This reinforces the idea that dislocations at the base of the whiskers play an important part in the mechanism, for typical fluid velocities in natural convection around a growing crystal of $0.1-1 \mathrm{~mm} \mathrm{~s}^{-1}-3$ to 4 orders of magnitude less than the forced convection velocity we considered above-would not break off a typical whisker unless the material strength were some orders of magnitude below the theoretical maximum. The nucleation of a crystal of one or other chirality is a symmetry-breaking event on the microscale, but the nonlinear autocatalytic dynamics of secondary nucleation amplify this to the macroscale, to the extent that an entire experiment may be influenced by the chirality of a single mother crystal: an ancestral Eve for the whole population.

We acknowledge the support of the Spanish Ministerio de Ciencia y Tecnología projects CONOCE, IMAGEN, and PPQ2001-2932.

[1] L. Pasteur, Compt. Rend. 26, 535 (1848).

[2] M. Avalos, R. Babiano, P. Cintas, J. L. Jiménez, and J. C. Palacios, Tetrahedron: Asymmetry 11, 2845 (2000).

[3] D. K. Kondepudi, R. J. Kaufman, and N. Singh, Science 250, 975 (1990).

[4] D. K. Kondepudi, J. Laudadio, and K. Asakura, J. Am. Chem. Soc. 121, 1448 (1999).

[5] K. Asakura, K. Kobayashi, Y. Mizusawa, T. Ozawa, S. Osanai, and S. Yoshikawa, Physica (Amsterdam) 84D, 72 (1995).

[6] K. Asakura, D. K. Kondepudi, and R. Martin, Chirality 10, 343 (1998).

[7] B. Martin, A. Tharrington, and X. 1. Wu, Phys. Rev. Lett. 77, 2826 (1996).

[8] T. Buhse, D. Durand, D. Kondepudi, J. Laudadio, and S. Spilker, Phys. Rev. Lett. 84, 4405 (2000).

[9] J. M. García-Ruiz, J. Struct. Biol. 142, 22 (2003).

[10] F. C. Frank, Biochim. Biophys. Acta 11, 459 (1953).

[11] G. Metcalfe and J. M. Ottino, Phys. Rev. Lett. 72, 2875 (1994).

[12] D. K. Kondepudi, K. L. Bullock, J. A. Digits, and P. D. Yarborough, J. Am. Chem. Soc. 117, 401 (1995).

[13] C. Viedma, J. Cryst. Growth 261, 118 (2004).

[14] R. F. Strickland-Constable, Kinetics and Mechanism of Crystallisation (Academic Press, London, 1968).

[15] J. Nývlt, O. Söhnel, M. Matuchová, and M. Broul, The Kinetics of Industrial Crystallization (Elsevier, Amsterdam, 1985).

[16] R.-Y. Qian and G. D. Botsaris, Chem. Eng. Sci. 53, 1745 (1998).

[17] A. A. Chernov, J. Struct. Biol. 142, 3 (2003).

[18] R. Philips, Crystals, Defects and Microstructures (Cambridge University Press, Cambridge, U.K., 2001).

[19] G. W. Sears, J. Chem. Phys. 26, 1549 (1957).

[20] D. K. Kondepudi and C. Sabanayagam, Chem. Phys. Lett. 217, 364 (1994).

[21] J. Szurgot and M. Szurgot, Cryst. Res. Technol.. 29, 829 (1994).

[22] M. Szurgot and J. Szurgot, Cryst. Res. Technol. 30, 949 (1995). 